\title{
Is Less Invasive Surfactant Administration Better than INtubation-SURfactant-Extubation for Prophylactic Surfactant Replacement Therapy?
}

Hyung-Joon Joo, MD and Gyu Hong Shim, MD, PhD

Department of Pediatrics, Inje University Sanggye Paik Hospital, Seoul, Korea

\section{ABSTRACT}

Purpose: The study aimed to examine whether prophylactic surfactant replacement therapy (SRT) with less invasive surfactant administration (LISA) by tracheal catheterization in a group of spontaneously breathing preterm infants would improve clinical outcomes compared to prophylactic SRT with the INtubation-SURfactantExtubation (INSURE) method.

Methods: We compared 20 spontaneously breathing preterm infants, 25 to 29 weeks of gestation or with a birth weight of less than $1,250 \mathrm{~g}$, treated with prophylactic SRT using a gastric tube (LISA group), to the 20 spontaneously breathing preterm infants matched by gestational age and birth weight, managed with prophylactic SRT via the INSURE method (INSURE group, historical control).

Results: The LISA group had lower rates of mechanical ventilation (MV) 72 hours after birth $(P=0.019)$ and at any time $(P=0.025)$, lower frequency of bradycardia during SRT $(P=0.031)$, and lower median duration of MV than the INSURE group $(P=0.038)$. In multivariate analysis, the LISA method was associated with a significantly lower likelihood of receiving invasive ventilation during hospitalization (odds ratio [OR], 0.029 ; 95\% confidence interval [CI], 0.001 to 0.938 ; $P=0.046)$ and a decreased frequency of bradycardia during SRT (OR, $0.020 ; 95 \% \mathrm{CI}, 0.001$ to $0.535 ; P=0.020$ ) as compared to the INSURE method.

Conclusion: Prophylactic SRT using LISA via tracheal catheterization in preterm infants may significantly reduce exposure to MV during hospitalization and brady. cardia during surfactant administration.

Key Words: Respiratory distress syndrome; Preterm infants; Pulmonary surfactant; Bradycardia

\section{INTRODUCTION}

Respiratory distress syndrome (RDS) is the leading cause of respiratory morbidity and
Received: 18 January 2022

Revised: 9 February 2022

Accepted: 15 February 2022

Correspondence to: Gyu Hong Shim, $\mathrm{MD}, \mathrm{PhD}$

Department of Pediatrics, Inje University Sanggye Paik Hospital, 1342 Dongil-ro, Nowon-gu, Seoul 01757, Korea

Tel: +82-2-950-1632

Fax: +82-2-950-1246

E-mail: peddoc@paik.ac.kr

Copyright(c) 2022 By Korean Society of Neonatology

This is an Open-Access article distributed under the terms of the Creative Commons Attribution Non-Commercial License (http:// creativecommons.org/licenses/by-nc/4.0), which permits unrestricted non-commercial use, distribution, and reproduction in any medium, provided the original work is pro perly cited. 
mortality in preterm infants ${ }^{1,2)}$. Previously, neonates with RDS were treated with surfactant replacement therapy (SRT) through endotracheal intubation and subsequent invasive mechanical ventilation (MV). SRT is an effective treatment for reducing pulmonary morbidities and mortality in neonates ${ }^{2}$. Prophylactic SRT could reduce respiratory morbidities and mortality compared to selective SRT, leading to widespread acceptance of prophylactic SRT as a form of management for neonatal RDS in the late 1990 s and the early $2000 \mathrm{~s}^{3)}$.

However, conventional SRT requiring endotracheal intubation and invasive ventilation is potentially harmful to the premature lungs, increasing the risk for acute injury ${ }^{4,5)}$. To overcome these problems, nasal continuous positive airway pressure (CPAP) is recognized as the suitable substitute for invasive ventilation in preterm infants ${ }^{6)}$.

However, optimal technique for administration of surfactants to the non-intubated neonates remains unknown ${ }^{7}$. Verder et al. ${ }^{8,9)}$ introduced the intubation-SURfactant-Extubation (INSURE) method to overcome CPAP-surfactant dilemma. However, this method has several problems, such as the need to use sedatives, increased risk for apnea, bradycardia, cardiopulmonary instability, and difficulty in extubation ${ }^{7)}$. To solve these problems, less invasive surfactant administration (LISA) by tracheal catheterization was introduced in spontaneously breathing preterm infants who underwent non-invasive ventilation, such as nasal CPAP, in the 1st week after birth ${ }^{10,11)}$. In recent studies, primary nasal CPAP and selective SRT with LISA by tracheal catheterization in preterm infants improved respiratory outcomes $^{12,13)}$. However, only a few studies have investigated prophylactic SRT using the LISA method. In this study, we administered "prophylactic" SRT by tracheal catheterization without endotracheal intubation in preterm infants who underwent nasal CPAP. We prospectively assessed the neonatal outcomes of prophylactic SRT by tracheal catheterization in preterm infants at 25 to 29 weeks of gestation or with a birth weight of less than $1,250 \mathrm{~g}$, and compared them to a historical cohort of preterm infants who initially received prophylactic SRT using the INSURE method.

\section{MATERIALS AND METHODS}

\section{Study population}

This single-center prospective cohort study was conducted in the neonatal intensive care unit (NICU) of the hospital over a 2 -year period. We included 20 preterm infants born at 25 to 29 weeks of gestation or with a birth weight of less than 1,250 g, who received prophylactic pulmonary surfactant $(200 \mathrm{mg} /$ kg Curosurf, Chiesi Pharmaceuticals, Parma, Italy) by tracheal catheterization (5-French gastric tube) while applying nasal CPAP without endotracheal intubation between January 1, 2015 and December 31, 2016 (LISA group). We compared them to the INSURE group (historical control) of 20 infants managed in our hospital with prophylactic surfactant treatment via the INSURE method between January 1, 2013 and December 31, 2014. The eligible INSURE group was matched to a case by gestational age (within \pm 7 days) and birth weight (within $\pm 250 \mathrm{~g}$ ). The inclusion criteria of the LISA and INSURE groups were: (1) preterm infants with 25 to 29 weeks of gestation or with a birth weight of less than 1,250 g; (2) preterm infants born in the hospital and admitted to the NICU; and (3) primary use of nasal CPAP (5 to 8 $\mathrm{cm} \mathrm{H}_{2} \mathrm{O}$, Infant Flow SiPAP, CareFusion, San Diego, CA, USA) for respiratory support. The exclusion criteria were: (1) infants who were transferred from another hospital; (2) infants who required persistent positive pressure ventilation (PPV) in the delivery room during initial resuscitation; (3) infants who were diagnosed with chromosomal abnormalities; and (4) infants who were diagnosed with multiple congenital anomalies. The LISA group was treated with a prophylactic surfactant through a sterile 5 -French gastric tube while applying nasal CPAP. This procedure was performed as described by Gopel et al. ${ }^{14)}$ study. The INSURE group was treated with prophylactic surfactant using the INSURE method. When $\mathrm{FiO}_{2} \geq 0.6, \mathrm{pH}<7.20$, and $\mathrm{PaCO}_{2}>65 \mathrm{~mm} \mathrm{Hg}$ or recurrent apnea requiring $\mathrm{PPV}$ or $\mathrm{CPAP}$ setting of $\mathrm{FiO}_{2} \geq 0.6$, peak end-expiratory pressure (PEEP) $>8 \mathrm{~cm}$ of $\mathrm{H}_{2} \mathrm{O}$ to maintain $\mathrm{SpO}_{2}$ $90 \%$ to $95 \%$, invasive ventilation was applied after endotracheal intubation. Infants were extubated and weaned to nasal CPAP when they had minimal or no signs of respiratory difficulty and MV settings of PIP $\leq 15 \mathrm{~cm} \mathrm{H}_{2} \mathrm{O}, \mathrm{PEEP} \leq 5 \mathrm{~cm} \mathrm{H}_{2} \mathrm{O}$, rate $\leq 20$ rate per min, and $\mathrm{FiO}_{2} \leq 0.3$. If the infant continued to require $\mathrm{FiO}_{2}$ $>0.4$ and $\mathrm{PEEP}>8 \mathrm{~cm} \mathrm{H}_{2} \mathrm{O}$ after $6 \mathrm{~h}$ of initial SRT to maintain $\mathrm{SpO}_{2}$ $90 \%$ to $95 \%$, the second dose of surfactant (100 mg/kg Curosurf) was administered by the LISA or INSURE method.

\section{Primary and secondary outcomes}

The primary outcomes of our study included the need for MV at 72 hours after birth or during hospitalization due to failure of noninvasive respiratory support, failure of the procedure on the first 
attempt, and requirement of an additional dose of surfactant. The secondary outcomesincluded the presence of bradycardia or reflux during SRT, MV duration, nasal CPAP duration, heated humidified high-flow nasal cannula (HHHFNC) duration, and hospital stay duration. Additionally, patent ductus arteriosus (PDA) requiring treatment, necrotizing enterocolitis (NEC, greater than grade 2) ${ }^{15)}$, intraventricular hemorrhage (IVH, higher than grade 2) ${ }^{16}$, cystic periventricular leukomalacia, retinopathy of prematurity $(\mathrm{ROP})^{17)}$, culture-proven sepsis, pulmonary hemorrhage, pneumothorax, death, bronchopulmonary dysplasia (BPD) ${ }^{18)}$, and composite outcomes of death or BPD were investigated.

\section{Data collection}

Clinical characteristics were compiled prospectively from the LISA group and retrospectively from the INSURE group. Demographic data, including gestational age, birth weight, type of delivery, sex, multiple births, maternal age, antenatal steroid use, oligohydramnios, premature rupture of membranes (PROMs $\geq 18$ hours), histologic chorioamnionitis, maternal diabetes, maternal hypertension, small for gestational age (SGA), Apgar score (1- and 5-minute), need for oxygen supplementation in the delivery room, and need for PPV support in the delivery room, were collected. Data on treatment during hospitalization were also collected. Thereafter, the results were compared between groups using statistical analysis.

\section{Statistical analysis}

Continuous variables were analyzed using Student's $t$-test or Mann-Whitney $U$-test. Categorical variables were analyzed using the chi-squared test or Fisher's exact test. All analyses indicated that the $P$-value was statistically significant at $P<0.05$. Continuous data are expressed as median (interquartile range [IQR]) or mean \pm standard deviation, and dichotomous data are expressed as number (\%). Additionally, multivariate logistic regression analysis was performed for all the statistically significant variables in the univariate analysis to adjust for the effect of LISA on known confounding variables (gestational age, SGA, sex, multiple births, and use of antenatal steroids). Statistical analysis was performed using the IBM SPSS software version 25.0 for Windows (IBM Co., Armonk, NY, USA).

\section{RESULTS}

\section{Study population}

From January 1, 2015 to December 31, 2016, 30 preterm infants, 25 to 29 weeks of gestation, or with a birth weight of less than $1,250 \mathrm{~g}$, were treated with prophylactic SRT. Five neonates

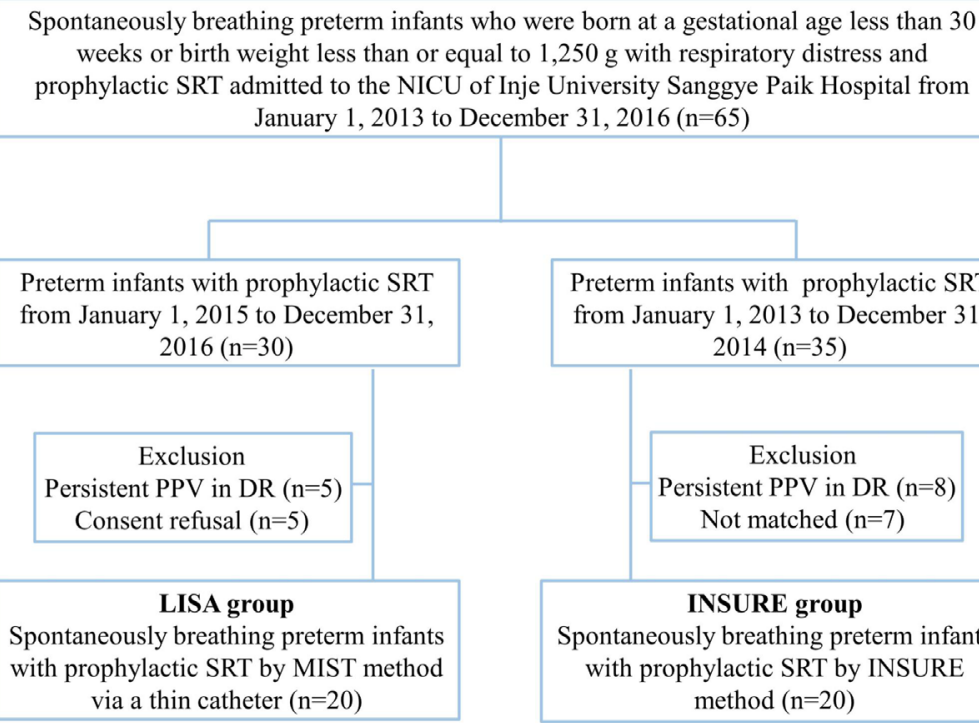

Preterm infants with prophylactic SRT from January 1, 2015 to December 31, $2016(\mathrm{n}=30)$

Exclusion

Persistent PPV in DR ( $\mathrm{n}=5$ )

Consent refusal $(n=5)$

LISA group

Spontaneously breathing preterm infants with prophylactic SRT by MIST method via a thin catheter $(n=20)$

Figure 1. Study population and subgrouping of prophylactic surfactant replace ment therapy with less invasive surfactant administration (LISA) (LISA group) and INtubation-SURfactant-Extubation (INSURE) method (INSURE group, historical control). Abbreviations: NICU, neonatal intensive care unit; SRT, surfactant replacement therapy; PPV, positive pressure ventilation; DR, delivery room; MIST, minimally invasive surfactant therapy. 
who required persistent PPV after birth and five who refused to provide consent to their parents, were excluded from the study. Twenty spontaneously breathing preterm infants managed with prophylactic SRT with the LISA method using a 5-French gastric tube were enrolled in the LISA group. From January 1, 2013, to December 31, 2014, 35 preterm infants, at 25 to 29 weeks of gestation, or with a birth weight of less than 1,250 g, were treated with prophylactic SRT via endotracheal intubation. Eight neonates who required persistent PPV after birth during initial resuscitation and seven neonates who did not match gestational age and birth weight, were excluded from this study. Twenty spontaneously breathing preterm infants who were treated with prophylactic SRT according to the INSURE method via an

Table 1. Baseline Demographic Data of the Study Population

\begin{tabular}{|c|c|c|c|}
\hline & $\begin{array}{c}\text { LISA } \\
\text { group } \\
(\mathrm{n}=20)\end{array}$ & $\begin{array}{c}\text { INSURE } \\
\text { group } \\
(n=20)\end{array}$ & $\begin{array}{c}P- \\
\text { value }\end{array}$ \\
\hline Gestational age (wk) & $28.3 \pm 1.2$ & $28.6 \pm 1.1$ & 0.562 \\
\hline Birth weight, $g$ & $1146 \pm 158$ & $1182 \pm 220$ & 0.562 \\
\hline Male & $11(55)$ & $11(55)$ & $>0.99$ \\
\hline Multiple birth & $5(25)$ & $3(15)$ & 0.695 \\
\hline Any antenatal steroids & $13(65)$ & $17(85)$ & 0.273 \\
\hline Cesarean section & $14(70)$ & $12(60)$ & 0.741 \\
\hline SGA & $1(5)$ & $2(10)$ & $>0.99$ \\
\hline Apgar at 1 minute & $4.9 \pm 1.3$ & $4.9 \pm 1.6$ & 0.917 \\
\hline Apgar at 5 minutes & $7.1 \pm 1.1$ & $7.1 \pm 1.3$ & 0.788 \\
\hline PROM $\geq 18$ hours & $5(25)$ & $11(55)$ & 0.105 \\
\hline Maternal age & $33.8 \pm 4.5$ & $32.8 \pm 2.8$ & 0.405 \\
\hline Histologic chorioamnionitis & $7(35)$ & $10(50)$ & 0.523 \\
\hline Oligohydramnios & $5(25)$ & $5(25)$ & $>0.99$ \\
\hline Maternal diabetes & $3(15)$ & $5(25)$ & 0.695 \\
\hline Maternal hypertension & $7(35)$ & $4(20)$ & 0.480 \\
\hline Oxygen supplement at delivery room & $15(75)$ & $15(75)$ & $>0.99$ \\
\hline PPV at birth & $6(30)$ & $7(35)$ & $>0.99$ \\
\hline $\begin{array}{l}\text { Time interval from birth to } \\
\text { surfactant administration ( } \mathrm{min} \text { ) }\end{array}$ & $28.2 \pm 7.5$ & $24.7 \pm 10.1$ & 0.196 \\
\hline Baseline $\mathrm{FiO}_{2}$ & $0.31 \pm 0.06$ & $0.32 \pm 0.07$ & 0.399 \\
\hline Baseline PEEP & $5.40 \pm 0.50$ & $5.27 \pm 0.45$ & 0.332 \\
\hline Baseline capillary CBGA pH & $7.29 \pm 0.08$ & $7.29 \pm 0.07$ & 0.887 \\
\hline Baseline capillary $\mathrm{CBGA} \mathrm{pCO}_{2}$ & $52.3 \pm 13.1$ & $55.5 \pm 12.4$ & 0.379 \\
\hline
\end{tabular}

Values are expressed as mean \pm standard deviation or number (\%). A $P<0.05$ represents statistically significant.

Abbreviations: LISA, less invasive surfactant administration; INSURE, INtubation-SURfactant-Extubation; SGA, small for gestational age; PROM, premature rupture of membrane; $\mathrm{PPV}$, positive pressure ventilation; $\mathrm{FiO}_{2}$, fraction of inspired oxygen; PEEP, peak end-expiratory pressure; CBGA, capillary blood gas analysis. endotracheal tube were enrolled in the INSURE group (Figure 1).

\section{Clinical characteristics}

Comparisons of the clinical characteristics between the two groups are shown in Table 1. There were no statistically significant differences between the groups in terms of gestational age, birth weight, type of delivery, sex, maternal age, multiple births, antenatal steroids use, maternal diabetes, maternal hypertension, SGA, oligohydramnios, PROM, histologic chorioamnionitis, Ap gar scores at -1 and 5 minute, oxygen supplementation or PPV in the delivery room, baseline concentration of inspired oxygen, and baseline capillary blood gas analysis.

\section{Primary and secondary outcomes}

In the univariate analysis, the LISA group had a significantly lower likelihood of receiving MV within 72 hours after birth $(P=0.019)$ or during hospitalization $(P=0.025)$ than the INSURE group. However, there were no significant differences in the second dose of surfactant use and failure on the first attempt using the LISA or INSURE method. The frequency of bradycardia during SRT was significantly lower in the LISA group than in the control group $(P=0.031)$. The median duration of MV ( 0 days vs. 6 days, $P=0.038$ ) was shorter in the LISA group than in the INSURE group. In contrast, the median duration of nasal CPAP, MV+nasal CPAP, HHHFNC, and MV+nasal CPAP+HHHFNC were not significantly different between the two groups (Table 2, Figure 2). In the multivariate analysis, prophylactic SRT with LISA was associated with a significantly lower likelihood of receiving MV during hospitalization (odds ratio [OR], 0.029; 95\% confidence interval [CI], 0.001 to $0.938 ; P=0.046$ ) and a decreased incidence of bradycardia during SRT (OR, 0.020 ; $95 \%$ CI, 0.001 to 0.535 ; $P=0.020$ ) as compared to the INSURE method.

There were no significant differences in neonatal morbidities (pneumothorax, PDA, NEC, ROP laser, IVH, culture-proven sepsis, BPD, mortality, composite outcome of BPD and mortality, and hospital stay) between the two groups (Table 3).

\section{DISCUSSION}

The purpose of our study was to evaluate whether prophylactic SRT by tracheal catheterization without endotracheal intubation in preterm infants who underwent nasal CPAP could be beneficial for preterm infants with prophylactic SRT using the 
Table 2. Primary and Secondary Outcomes

\begin{tabular}{|c|c|c|c|c|}
\hline & $\begin{array}{l}\text { LISA group } \\
\quad(n=20)\end{array}$ & $\begin{array}{l}\text { INSURE group } \\
(\mathrm{n}=20)\end{array}$ & $P$-value & $\begin{array}{c}\text { Adjusted OR }(95 \% \mathrm{CI}) \\
P \text {-value }\end{array}$ \\
\hline \multicolumn{5}{|l|}{ Primary outcomes } \\
\hline MV anytime & $8(40)$ & $15(75)$ & 0.025 & $0.029(0.001-0.938) ; 0.046$ \\
\hline Failure of procedure on the 1st attempt & $2(10)$ & $4(20)$ & 0.661 & \\
\hline Second dose of surfactant & $2(10)$ & $3(15)$ & $>0.99$ & \\
\hline Bradycardia during SRT & $2(10)$ & $9(45)$ & 0.031 & $0.020(0.001-0.535) ; 0.020$ \\
\hline Reflux during SRT & $5(25)$ & $9(45)$ & 0.320 & \\
\hline Duration of MV (d) & $0(0-2.75)$ & $6.00(1.25-14.25)$ & 0.038 & 0.919 (0.710-1.189); 0.520 \\
\hline Duration of nasal CPAP (d) & $10.00(4.00-14.00)$ & $7.50(5.00-11.75)$ & 0.767 & \\
\hline Duration of MV+nasal CPAP (d) & $13.50(4.00-17.50)$ & $16.50(10.25-23.75)$ & 0.116 & \\
\hline Hospital stays (d) & $64.00(52.00-75.50)$ & $68.50(53.00-79.00)$ & 0.258 & \\
\hline
\end{tabular}

Values are expressed as number (\%) or median (IQR). A $P<0.05$ represents statistically significant. Adjusted OR indicate the effect of SRT LISA versus INSURE were derived from multivariate logistic regression models including gestational age, small for gestational age, gender, multiple birth, antenatal steroids, SRT LISA or INSURE.

Abbreviations: LISA, less invasive surfactant administration; INSURE, INtubation-SURfactant-Extubation; OR, odds ratio; CI, confidence interval; MV, mechanical ventilation; SRT, surfactant replacement therapy; CPAP, continuous positive pressure ventilation; HHHFNC, heated humidified high-flow nasal cannula.

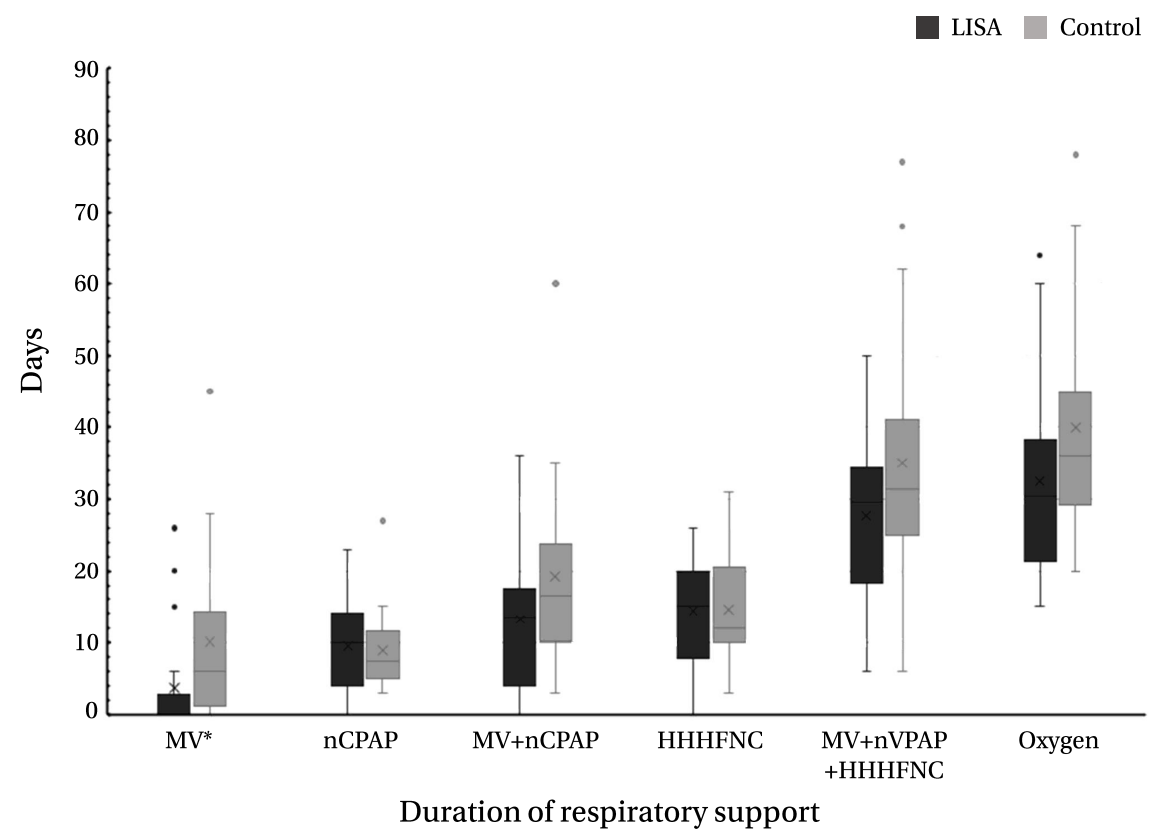

Figure 2. Median duration of mechanical ventilation (MV), nasal continuous positive airway pressure (nCPAP), heated humidified high-flow nasal cannula (HHHFNC), and oxygen uses. Values are expressed as median and interquartile range. *Statistically significant $(P<0.05)$. Abbreviation: LISA, less invasive surfactant administration. 
Table 3. Neonatal Morbidities and Respiratory Outcomes

\begin{tabular}{lccc}
\hline & $\begin{array}{c}\text { LISA group } \\
(\mathrm{n}=20)\end{array}$ & $\begin{array}{c}\text { INSURE group } \\
(\mathrm{n}=20)\end{array}$ & $P$-value \\
\hline PDA requiring treatment & $10(50)$ & $11(55)$ & $>0.99$ \\
NEC $\geq$ stage 2 & $1(5)$ & $3(15)$ & 0.605 \\
ROP laser & $2(10)$ & $3(15)$ & $>0.99$ \\
IVH $\geq$ stage 2 & $1(5)$ & $3(15)$ & 0.605 \\
Cystic PVL & $1(5)$ & $3(15)$ & 0.605 \\
Culture proven sepsis & $2(10)$ & $2(10)$ & $>0.99$ \\
Pneumothorax & 0 & $1(5)$ & $>0.99$ \\
Oxygen uses at 28 days & $10(50)$ & $12(60)$ & 0.525 \\
BPD & $4(20)$ & $6(30)$ & 0.716 \\
Death & $1(5)$ & $1(5)$ & $>0.99$ \\
BPD or death & $5(25)$ & $7(35)$ & 0.490
\end{tabular}

Values are expressed as number (\%). A $P<0.05$ represents statistically significant.

Abbreviations: LISA, less invasive surfactant administration; INSURE, INtubation-Surfactant-Extubation; PDA, patent ductus arteriosus; NEC, necrotizing enterocolitis; ROP, retinopathy of prematurity; IVH, intraventricular hemorrhage; PVL, periventricular leukomalacia; BPD, bronchopulmonary dysplasia.

INSURE method. To the best of our knowledge, this is the first prospective cohort study to compare LISA and INSURE methods for prophylactic SRT in spontaneously breathing preterm infants. Our study showed that prophylactic SRT using the LISA method in spontaneously breathing preterm infants, 25 to 29 weeks of gestation or with a birth weight of less than 1,250 g, could be used safely and reduce the frequency of MV exposure and bradycardia during surfactant administration compared to prophylactic SRT using the INSURE method.

Prophylactic SRT used in preterm infants showed decreased respiratory complications compared to the rescue surfactant treatment for RDS; however, elective use of invasive MV may cause adverse effects ${ }^{4}$. To avoid the effect of obligatory MV, primary nasal CPAP and INSURE methods were introduced as first-line treatments for preterm infants with respiratory distress to reduce acute lung injury ${ }^{5-9,19}$. However, how and when the surfactants should be administered remains unclear. In addition, the INSURE method has the problem of requiring endotracheal intubation and $\mathrm{PPV}^{7-9}$. To overcome these problems, including endotracheal intubation, PPV, and invasive MV, we believed that combining prophylactic SRT with the LISA method was one of the best ways. Thus, we planned this study to compare the combined prophylactic SRT and LISA methods in which both were known to improve respiratory prognosis.

The primary outcome of our study was the need for MV at
72 hours of birth or during hospital stay. Our study showed that three (15\%) infants in the LISA group received invasive ventilation versus $11(55 \%)$ in the INSURE group $(P=0.019)$ at 72 hours after birth. Eight (40\%) infants in the LISA group received invasive ventilation during hospitalization compared to the 15 (75\%) in the INSURE group $(P=0.025)$. As shown in previous studies, our study also showed that the proportion of MV needed at 72 hours after birth ${ }^{11,14,20)}$, or at any time ${ }^{21,22)}$ was significantly reduced in SRT by the LISA method compared to SRT by the INSURE method. In addition, the LISA group showed shorter median days on MV ( 0 day; IQR 0 to 2.8 ) as compared to the control group (6 days; IQR, 1.3 to 14.3 ) ( $P=0.038$ ). These results suggest that prophylactic SRT with the LISA method via a thin catheter may reduce the frequency and duration of invasive MV application compared to prophylactic SRT using the INSURE method. However, there were no significant differences in the median duration of MV+nasal CPAP or any respiratory support. Taken together, these results show that prophylactic SRT with the LISA method may reduce the duration of invasive MV, although additional studies are required to determine whether it could help to additionally reduce the total duration of respiratory support in neonates.

This study showed that the frequency of bradycardia during prophylactic SRT was significantly lower in the LISA group than that in the INSURE group. The frequency of bradycardia was lower than that reported by Dargaville et al. ${ }^{20,23)}$ (30\% to $45 \%)$, but was similar to the rates reported by Kanmaz et al. ${ }^{11)}$ (12\%), Bhattacharya et al. ${ }^{24)}(13 \%)$, and Kribs et al. ${ }^{25)}$ (17\%). It is not known why the frequency of bradycardia is relatively low in the LISA method. However, the relatively lower rates of bradycardia may be related to the lack of sedatives and avoidance of endotracheal intubation in all preterm infants. The sedatives used for endotracheal intubation may have caused decreased breathing, which can lead to secondary bradycardia. Bradycardia during endotracheal intubation could be caused by vagal nerve reflex due to direct stimulation of the pharynx and larynx. This type of bradycardia is called "unstable bradycardia," because it can easily disappear by temporarily suspending endotracheal intubation or sufficient oxygen supplement ${ }^{26)}$. This study suggests that the LISA method could reduce the occurrence of bradycardia because it does not require endotracheal intubation.

This study showed that there were no statistically significant differences in respiratory morbidities, namely BPD, pneumothorax, and mortality, between the groups. However, previous 
studies have shown that the LISA method using a thin catheter can reduce the prevalence of neonatal morbidities and mortality. Moreover, the LISA method via a thin catheter can reduce the incidence of $\mathrm{BPD}^{10,11,14,21,22)}$, pneumothorax ${ }^{27)}, \mathrm{NEC}^{28)}$, severe $\mathrm{IVH}^{25)}$, mortality ${ }^{25)}$, and the composite outcome of BPD or mortality ${ }^{21)}$. Two meta-analyses showed that SRT with the LISA method via a thin catheter may decrease BPD and the composite outcome of BPD or mortality ${ }^{12,29)}$. However, many previous studies have shown significant differences in gestational age, catheter type, and control groups. Therefore, additional studies are required to determine the optimal age and catheter type.

In our study, the side effects of prophylactic SRT using the LISA method were nothigher than those of the conventional methods. The theoretical advantages of prophylactic SRT through LISA include avoiding endotracheal intubation, minimizing PPV, and reducing the frequency of MV application to reduce MVinduce lung injury ${ }^{3-7)}$. Additionally, it can prevent a delay in the time required for the administration of pulmonary surfactants. If prophylactic SRT through LISA is confirmed to have fewer side effects, as in our current study, it could be considered as an alternative method to solve some problems, such as delay of SRT from nasal CPAP and selective SRT.

As shown in our study, the LISA method via a thin catheter has advantages over SRT with endotracheal intubation, including INSURE, but the exact mechanisms have not yet been clearly elucidated. However, the results of animal experiments and clinical trials have shown that the LISA method has the follow. ing theoretical advantages compared to SRT by endotracheal intubation: (1) prevention of lung injury induced by $\mathrm{PPV}^{30,31)}$; (2) reduction of tracheal trauma through the use of a small-diameter catheter for SRT ${ }^{32,33)}$; and (3) improvement of lung recruitment and aeration by maintaining spontaneous breathing ${ }^{34,35)}$.

This study had several limitations. First, the study was observational and exploratory in nature. The observational design is a barrier to causal conclusions; therefore, randomization studies are needed for further investigation. Second, the use of historical control limits the direct comparison between the two methods. It should be considered that there may be differences in the level of medical services according to different periods when using historical controls. Third, the study was a single-center pilot trial and the sample size was too small. Therefore, further prospective, large-scale, and multicenter trials are required. Fourth, some patients who did not need pulmonary surfactants were included in this study because all patients received prophylactic SRT via
LISA or INSURE. In addition, we did not include the nasal CPAP alone group in this study because of the widespread use of nasal CPAP. To overcome this problem, it is necessary to conduct a new study comparing prophylactic and selective SRT via the LISA method in spontaneously breathing preterm infants with nasal CPAP. Therefore, this is a pilot study on the possibility of prophylactic SRT using the LISA method in preterm infants who underwent nasal CPAP rather than prophylactic SRT using the INSURE method.

In conclusion, we observed that prophylactic SRT with the LISA method by tracheal catheterization in preterm infants, 25 to 29 weeks of gestation, or with a birth weight of less than $1,250 \mathrm{~g}$, could be used safely and may reduce the frequency of MV exposure at any time and bradycardia during surfactant administration.

\section{ARTICLE INFORMATION}

\section{Ethical statement}

This study was evaluated and approved by the Institutional Review Board of the Hospital, Seoul, Korea (approval number: SGPAIK 2014-12-025). This study was conducted with written consent from the parents.

\section{Conflicts of interest}

No potential conflict of interest relevant to this article was reported.

\section{Author contributions}

Conception or design: G.H.S.

Acquisition, analysis, or interpretation of data: H.J.J., G.H.S.

Drafting the work or revising: H.J.J., G.H.S.

Final approval of the manuscript: All authors read and approved the final manuscript.

\section{ORCID}

Hyung-Joon Joo https://orcid.org/0000-0003-3603-3093

Gyu Hong Shim https://orcid.org/0000-0002-0043-3186

\section{Funding}

None 


\section{Acknowledgments}

None

\section{REFERENCES}

1. Horbar JD, Badger GJ, Carpenter JH, Fanaroff AA, Kilpatrick S, LaCorte $\mathrm{M}$, et al. Trends in mortality and morbidity for very low birth weight infants, 1991-1999. Pediatrics 2002;110(1 Pt 1):14351.

2. Seger N, Soll R. Animal derived surfactant extract for treatment of respiratory distress syndrome. Cochrane Database Syst Rev 2009;2:CD007836.

3. Soll RF, Morley CJ. Prophylactic versus selective use of surfactant for preventing morbidity and mortality in preterm infants. Cochrane Database Syst Rev 2000;2:CD000510.

4. Attar MA, Donn SM. Mechanisms of ventilator-induced lung injury in premature infants. Semin Neonatol 2002;7:353-60.

5. Sweet DG, Carnielli V, Greisen G, Hallman M, Ozek E, Plavka R, et al. European consensus guidelines on the management of neonatal respiratory distress syndrome in preterm infants: 2013 update. Neonatology 2013;103:353-68.

6. Committee on Fetus and Newborn; American Academy of Pediatrics. Respiratory support in preterm infants at birth. Pediatrics 2014;133:171-4.

7. Aguar $M$, Vento $M$, Dargaville PA. Minimally invasive surfactant therapy: an update. NeoReviews 2014;15:e275-85.

8. Verder H, Robertson B, Greisen G, Ebbesen F, Albertsen P, Lundstrom K, et al. Surfactant therapy and nasal continuous positive airway pressure for newborns with respiratory distress syndrome. Danish-Swedish Multicenter Study Group. N Engl J Med 1994;331:1051-5.

9. Verder H, Albertsen P, Ebbesen F, Greisen G, Robertson B, Bertelsen A, et al. Nasal continuous positive airway pressure and early surfactant therapy for respiratory distress syndrome in newborns of less than 30 weeks' gestation. Pediatrics 1999; 103: E24.

10. Kribs A, Hartel C, Kattner E, Vochem M, Kuster H, Moller J, et al. Surfactant without intubation in preterm infants with respiratory distress: first multi-center data. Klin Padiatr 2010; 222:13-7.

11. Kanmaz HG, Erdeve O, Canpolat FE, Mutlu B, Dilmen U. Surfactant administration via thin catheter during spontaneous breathing: randomized controlled trial. Pediatrics 2013;131: e502-9.

12. Rigo V, Lefebvre C, Broux I. Surfactant instillation in spontaneously breathing preterm infants: a systematic review and meta-analysis. Eur J Pediatr 2016;175:1933-42.

13. Lau C, Chamberlain RS, Sun S. Less invasive surfactant administration reduces the need for mechanical ventilation in preterm infants: a meta-analysis. Glob Pediatr Health 2017; 4:2333794X17696683.

14. Gopel W, Kribs A, Ziegler A, Laux R, Hoehn T, Wieg C, et al. Avoidance of mechanical ventilation by surfactant treatment of spontaneously breathing preterm infants (AMV): an openlabel, randomised, controlled trial. Lancet 2011;378:1627-34.

15. Kliegman RM, Walsh MC. Neonatal necrotizing enterocolitis: pathogenesis, classification, and spectrum of illness. Curr Probl Pediatr 1987;17:213-88.

16. Papile LA, Burstein J, Burstein R, Koffler H. Incidence and evolution of subependymal and intraventricular hemorrhage: a study of infants with birth weights less than 1,500 gm. J Pediatr 1978;92:529-34.

17. International Committee for the Classification of Retinopathy of Prematurity. The International Classification of Retinopathy of Prematurity revisited. Arch Ophthalmol 2005;123:991-9.

18. Jobe AH, Bancalari E. Bronchopulmonary dysplasia. Am J Respir Crit Care Med 2001;163:1723-9.

19. Schmolzer GM, Kumar M, Pichler G, Aziz K, O'Reilly M, Cheung PY. Non-invasive versus invasive respiratory support in preterm infants at birth: systematic review and meta-analysis. BMJ 2013; 347:f5980.

20. Dargaville PA, Aiyappan A, De Paoli AG, Kuschel CA, Kamlin $\mathrm{CO}$, Carlin JB, et al. Minimally-invasive surfactant therapy in preterm infants on continuous positive airway pressure. Arch Dis Child Fetal Neonatal Ed 2013;98:F122-6.

21. Gopel W, Kribs A, Hartel C, Avenarius S, Teig N, Groneck P, et al. Less invasive surfactant administration is associated with improved pulmonary outcomes in spontaneously breathing preterm infants. Acta Paediatr 2015;104:241-6.

22. Krajewski P, Chudzik A, Strzalko-Gloskowska B, Gorska M, Kmiecik M, Wieckowska K, et al. Surfactant administration without intubation in preterm infants with respiratory distress syndrome: our experiences. J Matern Fetal Neonatal Med 2015;28:1161-4.

23. Dargaville PA, Aiyappan A, Cornelius A, Williams C, De Paoli AG. Preliminary evaluation of a new technique of minimally invasive surfactant therapy. Arch Dis Child Fetal Neonatal Ed 2011;96:F243-8.

24. Bhattacharya S, Read B, McGovern E, da Silva O. Highvolume surfactant administration using a minimally invasive technique: experience from a Canadian neonatal intensive care unit. Paediatr Child Health 2019;24:313-7.

25. Kribs A, Pillekamp F, Hunseler C, Vierzig A, Roth B. Early administration of surfactant in spontaneous breathing with nCPAP: feasibility and outcome in extremely premature infants (postmenstrual age $</=27$ weeks). Paediatr Anaesth 2007;17: 364-9.

26. Jones P, Dauger S, Peters MJ. Bradycardia during critical care 
intubation: mechanisms, significance and atropine. Arch Dis Child 2012;97:139-44.

27. Kribs A, Roll C, Gopel W, Wieg C, Groneck P, Laux R, et al. Nonintubated surfactant application vs conventional therapy in extremely preterm infants: a randomized clinical trial. JAMA Pediatr 2015;169:723-30.

28. Mirnia K, Heidarzadeh M, Hoseini MB, Sadeghnia A, Akrami F, Balila $\mathrm{M}$, et al. Surfactant administration via thin catheter during spontaneous breathing: randomized controlled trial in Alzahra hospital. Iran J Neonatol 2013;4:5-9.

29. Aldana-Aguirre JC, Pinto M, Featherstone RM, Kumar M. Less invasive surfactant administration versus intubation for surfactant delivery in preterm infants with respiratory distress syndrome: a systematic review and meta-analysis. Arch Dis Child Fetal Neonatal Ed 2017;102:F17-23.

30. Niemarkt HJ, Kuypers E, Jellema R, Ophelders D, Hutten M, Nikiforou M, et al. Effects of less-invasive surfactant administration on oxygenation, pulmonary surfactant distribution, and lung compliance in spontaneously breathing preterm lambs. Pediatr Res 2014;76:166-70.

31. Ricci F, Bresesti I, LaVerde P, Salomone F, Casiraghi C, Mersanne A, et al. Surfactant lung delivery with LISA and InSurE in adult rabbits with respiratory distress. Pediatr Res 2021;90:576-83.

32. Vento M, Bohlin K, Herting E, Roehr CC, Dargaville PA. Sur factant administration via thin catheter: a practical guide. Neonatology 2019;116:211-26.

33. Herting E, Hartel C, Gopel W. Less invasive surfactant administration: best practices and unanswered questions. Curr Opin Pediatr 2020;32:228-34.

34. Bohlin K, Bouhafs RK, Jarstrand C, Curstedt T, Blennow M, Robertson B. Spontaneous breathing or mechanical ventilation alters lung compliance and tissue association of exogenous surfactant in preterm newborn rabbits. Pediatr Res 2005;57(5 Pt 1):624-30.

35. van der Burg PS, de Jongh FH, Miedema M, Frerichs I, van Kaam AH. Effect of minimally invasive surfactant therapy on lung volume and ventilation in preterm infants. J Pediatr 2016;170:67-72. 